\title{
Kants Lehre vom höchsten Gut.
}

\author{
Eine Richtigstellung.
}

Von A. Döring.

Im Oktober and November 1895 warde in Zweigverein des Evangelischen Bundes in Berlin ein Cyklus apologetischer Vorträge gehalten, die auch nachber in Broschürenform im Drucke erschienen sind. $\mathrm{Za}$ dieser Serie gehört anch der Vortrag von Kaftan: Das Christentam and die Philosophie (Leipzig 1896). Es ist nicht dieses Ortes and liegt auch nicht in meiner Absicht, die gesamte Argamentation dieses Vortrages einer Beurteilung za unterwerfen. ${ }^{2}$ ) Dagegen scheint mir die Art, wie hier für die Lehre vom höchsten Gat als Central-Dogma der Philosophie und für die Wesensbestimmung des höchsten Gates die Autorität Kants in Anspruch genommen wird, anch hente noch einer Richtigstellang zu bedürfen, zumal anch von der neueren Kantforschung die hiermit zusammenhängende, für den Aufban des Kantischen Systems so wichtige Gedankenreihe vielfach unbillig vernachlässigt worden ist.

Wir hören nämlich in diesem Vortrage, die Philosophie sei nach Aristoteles , die Wissenschaft von den letzten Gründen oder den ersten Ursachen alles Seienden", nach Kant dagegen die Lehre vom höchsten Gat (S. 5), mit welcher letzteren Begriffsbestimmang anch der Vortragende seinerseits sich einverstanden erklärt (S. 15). Es wird dann ferner (S. 22) behauptet, Plato habe das höchste Gut in die Erkenntnis, Kant dagegen in "das sittliche Wollen and Handeln" gesetzt. Mit dieser Fassung babe Kant zaerst den Grundgedanken der Reformation za einem philosophischen Prinzip erhoben and verdiene daram der Philosoph des Protestantismus za heissen. Ebenso heisst es S. 24 f., die „ethisch bedingte Lehre vom höchsten Gute" sei der eigentliche and letzte Schliussel des Weltverständnisses, und die wahre Philosophie, die diesen Weg fubre, sei diejenige, die sich wesentlich in den Bahnen Kants bewege.

1) Die „Kantstudien“ haben über den Kaftanschen Vortrag im I. Band, S. 284 eingehender berichtet.

(Anmerkung der Redaktion.) 
Es wird also hier Kant für folgende zwei Sätze als Autorität herangezogen:

1. Die Philosophie ist die Lehre vom höchsten Gate.

2. Das höchste Gat besteht im sittlichen Wollen and Handeln.

Ich weiss nicht, ob Kaftan an anderer Stelle diese Interpretation Kants eingebender begrundet hat. Jedenfalls bertuhrt es befremdlich, den ingrimmigsten Gegner des Eudämonismus in jeder Form bier sans façon selbst zum Eudämonisten gestempelt zu sehen, and es darf nicht unwidersprochen bleiben, wenn in einem für das grössere Pablikam bestimmten Vortrage Kant fur einen an sich durchaus berechtigten, aber seiner Denkweise völlig widerstrebenden Gedanken als Autorität angerafen wird. Ich beabsichtige jedoch nicht, für die allerdings sehr interessante Frage das etwas weitschichtige Material in extenso vorzufubren, sondern werde mich anf die allerwesentlichsten Punkte beschränken.')

\section{Kants Lehre von der Aufgabe der Philosophie.}

Die centrale Stellang der Lehre vom höchsten Gate in der Philosophie bildet, wie Kant selbst an mebreren nachher anzufuhrenden Stellen hervorgehoben hat, das Charakteristikum der antiken Philosophie in der Zeit nach Plato and Aristoteles. Der Übergang dazu ist schon im Alterssystem Platos, dentlicher noch bei Aristoteles za beobachten. Das volle Bewusstsein dieser Sachlage beherrscht die nacharistotelischen Systeme, das akademische, peripatetische, stoische and epikareische.

Über seine eigene Auffassung vom Wesen und der Aufgabe der Philosophie spricht sich Kant zuerst in der Kr. d. r. V. in der „Architektonik" aus. Er unterscheidet hier den Schulbegriff and den Weltbegriff der Philosophie. Der Schulbegriff bezieht sich lediglich auf die Erkenntnisart. Die empirische oder, wie Kant sagt, historische Erkenntnisart ist Erkenntnis ex datis, die rationale dagegen Erkenntnis ex principiis. Diese zerfällt wieder in die ans der Konstruktion der Begriffe, die Mathematik, und die ans Begriffen schlechthin, die Philosophie.

Der Weltbegriff einer Wissenschaft überhanpt ist „derjenige, der das betrifft, was jedermann notwendig interessiert." Dies auf

1) Vgl. meine Sohrift „Über den Begriff der Philosophie“ (Dortmund 1878) S. $36 \mathrm{ff}$., ferner meinen Aufsatz in den Preufs. Jahrbüchern „Über Kants Lehre von Begriff und Aufgabe der Philosophie" (Band 56, 1885), sowie endlich anch meine „Philosophische Güterlehre" (S. 899 ff.). 
die Philosophie angewandt, ergiebt die Definition derselben als die Wissenschaft von der Beziehung aller Erkenntnis anf die wesentlichen Zwecke der menschlichen Vernunft." Diese wesentlichen Zwecke aber mussen sich bei vollkommener systematischer Einheit der Vernunft wieder auf einen einzigen reduzieren. Es entsteht der Unterschied vom Endzweck und subalternen Zwecken, die sich zu jenem als Mittel verbalten. Über die Natar des Endzweckes spricht sich Kant an dieser Stelle nur summarisch aus: Der erstere ist kein anderer, als die ganze Bestimmung des Menschen und die Philosophie uber dieselbe beisst Moral" (Ros. S. 644 ff.).

Genaueres uber das Verbältnis dieser Zwecke erfahren wir im 1. Abschnitt des "Kanons" „Von dem letzten Zwecke des reinen Gebrauchs unserer Vernunft" (Ros. 615 ff.). Hier werden als die letzten Zwecke der Vernunft bezeichnet Freiheit, Unsterblichkeit und Dasein Gottes. Das theoretische Interesse an diesen drei Problemen aber ist gering; ibre eigentliche Bedeutung gewinnen sie erst durch die praktische Frage, "was zu thun sei, wenn der Wille frei, wenn ein Gott und eine kunftige Welt ist". Somit ist „die letzte Absicht der weislich uns versorgenden Natur bei der Einrichtung unserer Vernunft eigentlich nur aufs Moralische gestellet". „Die höchsten Zwecke sind die der Moralität" (Ros. 630).

Wir bemerken hier, wie vorsichtig Kant jeder Beziehung auf Glückseligkeit ans dem Wege geht. Moralität ist nicht das Mitiel zar wahren Glückseligkeit, sondern „die Bestimmung des Menschen“. Dieser haltlose, nicht weiter gepriifte und analysierte Begriff verrät ihn als Sobn seiner Zeit. Der religiös-sittliche Rigorismus des Pietisnus ist in den rein moralischen Rigorismus des Aufklärungszeitalters ubergegangen. Bemerkenswert ist hierbei, wie Kant, der abgesagte Feind der Heteronomie, gerade im obersten Begriffe seines Gedankensystems der Heteronomie anheimfällt. Er wagt noch nicht den Schritt, das Individuum autonom sein wabres Wohlsein, seine Befriedigung suchen zu lassen. Über ihm schwebt gebieterisch seine „Bestimmung."

In der That ist dies aber der oberste Begriff des ganzen Systems. Die ganze theoretische Philosophie gipfelt in der Frage nach den drei Ideen, d. h. nach den drei subalternen Zwecken, die zunächst berufen scheinen, den Endzweck zu stutzen. Das Resultat ist ein negatives. Aber das braucht in Bezug auf die Erreichung des Endzweckes keine Beunrubigung. żu gewähren. Denn einesteils wurde durch den Erweis ron Gott und Unsterblichkeit in Wirklich- 
keit die Bestimmang nicht erreicht; der theoretische Erweis wurde nur za einer sklarischen, heteronomen Legalität, keineswegs aber zu wahrer Moralität fuhren. Freiheit aber wäre za dieser Legalität nur ,im praktischen Verstande" (Kos. 618 f.) erforderlich. Andernteils bedarf aber die Erreichung der Bestimmung auch gar keiner theoretischer Hilfeleistung, da sie in der Thatsache des kategorischen Imperativs in absolut befriedigender Weise gewährleistet ist.

Die Erreichung der drei „wesentlichen" oder ,höchsten" Zwecke der Vernunft ist also 1. unmöglich, 2. wertlos und 3. für den Endzweck überfluissig and entbehrlich. Das ist das Kantsche System in nuce, selbstverständlich in derjenigen apophthegmatischen Fassung, die ich mir für diese Arbeit überhaupt zum Gesetze gemacht habe.

Dals nun die hier nach der Kr. d. r. V. entwickelte Begriffsbestimmung der Philosophie nicht etwa nur ein gelegentlicher Einfall Kants, sondern die seiner ganzen Denkrichtung und seinem Gedankenkreise konforme ist, ergiebt sich aufser dem soeben gelieferten inneren Nachweise auch noch durch äufsere Zengnisse. Die ganze Gedankenreihe der Erkenntnisse ex datis and ex principiis, des Schulbegriffs und Weltbegriffs, der letzten Zwecke und des Endzwecks der Vernunft findet sich wiederholt in der 1800 nach Kants Vorlesungsnotizen von Jäsche herausgegebenen Logik (Abschnitt U der Einleitung), sowie in etwas kürzerer Formulierung in den „Fortschritten der Metaphysik" (Ros. I. S. 488 ff.). Ich begnüge mich der Kürze halber hier damit, auf diese Stellen hinzuweisen. Eine dentliche Anspielung auf diese Begriffsbestimmung der Pbilozophie findet sich endlich auch noch in der letzten der von Kant selbst herausgegebenen Schriften, dem „Streit der Fakultäten“ (1798). Hier heisst es (Ros. X, 368), die Philosophie babe ihr Interesse am Ganzen des Endzweckes der Vernunft, der eine absolute Einbeit sei.

Die eigentliche Meinung Kants von der Aufgabe der Philosophie hängt also nicht an dem (in Wirklichkeit den Menschen zur Antonomie erhebenden) Begriffe des höchsten Gates, sondern an dem (heteronomen) Begriffe der „Bestimmung des Menschen“.

\section{Kants Lehre vom höchsten Gute.}

In der Kr. d. pr. V. verfolgt Kant zunächst das Interesse, das praktische Gesetz als ein von jeder sonstigen Beziehung abgelöstes. ausschlielslich aus reiner Vernunft geltendes (d. h. thatsächlich als ein Gesetz der praktischen Widersprachsfreiheit) za formalieren. In diesem Zusammenhange finden sich (Ros. 185 ff.) folgende Aus- 
fubrangen. Alle "Verirrangen der Philosophen in Ansebang des obersten Prinzips der Moral" haben darin ibren Grand, dass sie, statt zuerst nach einem a priori den Willen bestimmenden Gesetze za forschen, einen Gegenstand des Willens als Bestimmungsgrund desselben aufsuchten. Dadurch entsteht Heteronomie, mag dieser den Willen bestimmende Gegenstand als Gluckseligkeit, als Vollkommenheit, als moralisches Gesetz (hier ist wohl das Hutche s onsche Wohlgefallen am moralischen Verhalten gemeint, s. die Tafel der materialen praktischen Bestimmungsgrunde Ros.154) oder als Wille Gottes bestimmt werden. „Die Alten verrieten indessen diesen Febler dadurch unverbolen, dass sie ibre moralische Untersuchung gänzlich auf die Bestimmung des Begriffes $\nabla 0 \mathrm{~m}$ höchsten Gut, mithin eines Gegenstandes setzten, welchen sie nachher zum Bestimmungsgrande des Willens im moralischen Gesetze zu machen gedachten ... Die Neueren, bei denen die Frage über das höchste Gat ausser Gebrauch gekommen, zum wenigsten nur Nebensache geworden za sein scheint, verstecken obigen Fehler (wie in vielen anderen Fällen) hinter unbestimmten Worten", während doch auch bei ihnen ein a priori gehietendes moralisches Gesetz nicht zustande kommt.

Hier haben wir die echte unverfälschte Ansicht Kants vom höchsten Gat. Sie begründet in seinem Sinne Heteronomie des Sittengesetzes, wenn gleich, wie wir gesehen haben, thatsächlich Autonomie des Menschen.

Eine wesentlich veränderte Stellang zur Frage tritt jedoch in der praktischen Kritik da ein, wo das Interesse in den Vordergrund tritt, aus dem praktischen Gesetze die Postulate abzuleiten. In diesem Interesse erklärt Kant jetzt (Ros. S. 243 f.), die reine praktische Vernunft suche die Totalität ihres Gegenstandes unter dem Namen des böchsten Gates, und findet, dals die wissenschaftliche Bestimmung dieser Idee (des höchsten Gutes) als Maxime unseres vernünftigen Verbaltens das Wesen der Philosophie ansmache, in der Bedeatung, wie die Alten das Wort verstanden, nämlich als Anweisung zar richtigen Erfassung des Begriffs des höchsten Gutes and zum richtigen Verhalten behufs seiner Erwerbung. „Es wäre gat, wenn wir dieses Wort (Philosophie) bei seiner alten Bedeutang liessen, als eine Lehre vom höchsten Gut, sofern die Vernunft bestrebt ist, es darin zar Wissenschaft za bringen." $\mathrm{Er}$ geht sodann dazu uber (Ros. 245 f.), in der bekannten Weise den Begriff des höchsten Gutes $\mathrm{za}$ bestimmen: Tugènd als Gluckseligkeitswürdigkeit ist das oberste Gut, wie ,in der Analytik bewiesen 
worden" sei (diesen Beweis hat er in der Analytik nicht gefuihrt, konnte ihn auch gar nicht fuhren wollen, da er der ganzen Denkrichtung der Analytik schnurstracks zawiderläuft), ferner Gluckseligkeit als Ergänzung der Wurdigkeit zar Totalität. Bekanntlich entspringt aus dieser Wendang der Gedanken das Gottespostalat. Dieser selbe Gedankengang findet sich aber anch schon, zwar in minder entwickelter Fassung, aber doch auch wieder mit einigen eigentümlichen Zügen, in der Kr. d. r. V. im Abschnitte „Kanon“. Hier wird (Ros. 624) die Gottheit als eine solche Intelligenz, in der „der moralisch vollkommene Wille mit der höchsten Seligkeit verbunden" ist, das Ideal des höchsten Gutes, d. h. offenbar die ideale Verwirklichung desselben genannt, und in diesem Ideal des höchsten ursprünglichen Gates zugleich der Grand der „Verknüpfung der heiden Elemente des höchsten abgeleiteten" (d. h. dem endlichen Vernunftwesen zugänglichen) Gates postaliert.

Kant ist nicht der Meinung, in den beiden angefuihrten Stellen der praktischen Kritik Widersprechendes aufgestellt za haben. Er hat bei der Abfassung der ersten den zweiten Gedanken schon im Auge gehabt. Er bemerkt nämlich an ersterer Stelle, dass später, „wenn das moralische Gesetz allererst für sich bewährt und als unmittelbarer Bestimmangsgrand des Willens gerechtfertigt" sei, die Vorstellung eines Gegenstandes des Willens ihre Stelle finden werde. Dennoch ist dieser Widersprach thatsächlich vorhanden, was schon darin seinen Ausdrack findet, dass er an ersterer Stelle die Voranstellung des höchsten Gates als fundamentalen Fehler brandmarkt, an letzterer aber die Begriffsbestimmung sogar der gesamten Philosophie als Lehre vom höchsten Gut acceptiert.

Die Sache liegt also nach den bisherigen Feststellangen so, dass zunächst der erste der beiden oben formulierten Kaftanschen Sätze (Philosophie = Lebre vom höchsten Gut) mit einem Scheine des Rechts anf Grand einer einzigen Stelle Kant zageschrieben werden kann, dass aber diese Stelle sowohl mit seiner eigentlichen, unter I entwickelten Lehre über das Wesen der Philosophie, als anch mit seiner unter II vorangestellten Lehre von der Begrindung der Ethik in Widerspruch steht. Und was sodann den zweiten der Kattanschen Sätze (das höchste Gut bestebt nach Kant im sittlichen Wollen und Handeln) betrifft, so wird dieser auch nicht einmal durch diese einzige Stelle gewährleistet. Denn nach ihr ist die Tugend als Gluckseligkeitswürdigkeit zwar das oberste Gat, aber noch keineswegs der Gesamtinbegriff des böchsten Gates.

Es giebt nun allerdings zwei Stellen, an denen Kant eine der 
Kaftanschen Interpretation gunstige Begriffssynthese zwischen seiner Lebre vom Endzweck and vom höchsten Gate vollzieht. Die eine steht in den "Fortschritten der Metaphysik" (Ros. I, 532). Nach dieser Stelle ist der Endzweck der reinen praktischen Vernuntt das höchste Gat, bestehend in der Tugend als böchstem Erfordernis und Bedingung, und der binzutretenden Gluckseligkeit. Die zweite Stelle vollzieht die Synthese zwischen Endzweck und höchstem Gute ausdrucklich nur in der Überschrift des in Betracht kommenden Abschnitts. Der 2weite Abschnitt des Kanons der reinen Vernunft (Kr. d. r. V. Ros., S. 620) ist nämlich tiberschrieben: „Von dem Ideal des höchsten Guts als einem Bestimmungsgrunde des letyten $\mathrm{Z}$ wecks der reinen Vernunft." Was Kant unter dem Ideal der reinen Vernunft versteht, haben wir schon oben gesehen. Ebenso ist dort auch schon der Grandgedanke dieses Abschnittes angedentet worden, darin bestehend, dass die Gottheit als Ideal des höchsten Gutes zugleich als der Grund der Verknüpfung der Würdigkeit and Glückseligkeit bei den endlichen Vernunftwesen, also der Realisierung des abgeleiteten höchsten Gutes postaliert werden mass. Was in dieser Überschrift nea und eigentümlich ist, ist nur die Synthese dieses Verknüpfungsgedankens mit der Lehre rom Endzwecke der Vernunft. Gott als das Prinzip dieser Verknüpfung soll damit zugleich den "Bestimmangsgrand" des Endzweckes; d. b. doch wohl den Bestimmungsgrand für die Verfolgung desselben als Endzweckes abgeben.

Beide Stellen kommen also darin überein, den Endzweck, nämlich die Erfüllung der moralischen Bestimmung, in einen engen $\mathrm{Zu}$ sammenhang mit der Realisierang des höchsten Gutes za setzen, d. b. thatsächlich den Endzweck seiner absoluten Würde za entkleiden und von Glückseligkeitsfolgen abhängig za machen. Die erste Stelle identifiziert den Endzweck geradeza mit dem höchsten Gute, verfällt also uneingeschränkt in den in der praktischen Analytik gerügten Fehler der antiken Philosophie. Die zweite Stelle erklärt wenigstens die Überzengung vom Dasein der Gottheit fiir den Bestimmungsgrund zar Verfolgung des Endzweckes and tritt damit wenigstens in Widerspruch zu der Lehre vom kategorischen Imperativ als der unbedingt sicheren Gewähr für die Möglichkeit, die moralische Bestimmung zu erfüllen.

Ich kann in diesen beiden Stellen nur einen Beweis für die auch sonst bekannte Eigentümlichkeit Kants erblicken, teils scharf gezogene Begriffslinien nachträglich in einer gewissen Unachtsamkeit 
wieder zu verwischen, teils die gewonnenen scharfen Resultate seines Denkens nachträglich in einer gewissen Furchtsamkeit wieder abzaschwächen und den hergebrachten anzunähern, keineswegs aber kann ich darin verwertbare Zengnisse fur den eigentlichen Sinn seiner Lehre sehen. Wir mussen, um den eigentlichen Kant zu finden, uberall mit den beiden eben gekennzeichneten Eigentümlichkeiten seiner Darstellungsweise rechnen.

Schliesslich sei noch darauf aufmerksam gemacht, dass der von Kaftan unberechtigter Weise Kant beigelegte Standpunkt im wesentlichen der von mir in meiner "Philosophischen Guterlehre“ (Berlin 1888) vertretene ist. Ich vertrete in dieser Schrift einesteils den Satz, dass die Philosophie ihrem Wesen nach Gtiterlehre = Lehre vom höchsten Gate ist, andernteils zwar nicht den Satz, dass das höchste Gat unmittelbar im sittlichen Wollen and Handeln selbst besteht, wohl aber den Satz, dass die ant wahrem, nur durch sittliches Wollen and Handeln zu erwerbendem Eigenwert berahende berechtigte Selbstschätzang das höchste Gat ist. 